DOI: $10.15393 /$ j3.art.2019.5910

UDC 517.28, 517.958, 517.23

\author{
V. Sadhasivam, M. DeEpa
}

\title{
OSCILLATION CRITERIA FOR FRACTIONAL IMPULSIVE HYBRID PARTIAL DIFFERENTIAL EQUATIONS
}

\begin{abstract}
In this paper, we study the oscillatory behavior of the solutions of fractional-order nonlinear impulsive hybrid partial differential equations with the mixed boundary condition. By using the integral averaging method and the Riccati technique, we have obtained the oscillation criteria of all the solutions of the given system. An example is given to illustrate our main results.
\end{abstract}

Key words: Oscillation, Fractional partial differential equation, Hybrid differential equation, impulse.

2010 Mathematical Subject Classification: 34K34, 35B05, 35R11, 35R12.

1. Introduction In the last few decades, there has been a lot of interest in deriving sufficient conditions for the oscillation and nonoscillation of solutions of classes of differential equations. The first investigation and publication of the oscillation theory of impulsive differential equations was in 1989 [9], and the first paper on impulsive partial differential equations was published in 1996 [2]. Its consequences were included in the book [15]. Chatzarakis et al. [3], Kalaimani et al. [13], Prakash et al. [20], Sadhasivam et al. [23,25] and Yang et al. [30] studied the impulsive partial differential equations. The study of impulsive partial differential equations is motivated by various applications in population models [2], single species growth [6], feedback control prey-predator model [12], and various scientific models [29,31].

Even though there is a countable number of papers on oscillatory solutions of fractional partial differential equations, refer [7,16,21,22,24], they have not dealt with the impulse effect. Many authors have investigated some of the areas of applications of fractional differential equations, like viscoelasticity, electrochemistry, signal processing and so on; in the last few decades, there have been several monographs of fractional derivatives

(c) Petrozavodsk State University, 2019 
and integrals, see $[1,4,14,18,26,33]$ and the references cited therein. In the recent years, there has been much research on hybrid differential equations that refer to various aspects of quadratic perturbations of nonlinear differential equations. The reason is that hybrid differential equations include several dynamic systems as special cases. There has been a significant amount of work on the theory of hybrid differential equations that can be referred in articles $[10,27,28,32]$. Applications with numerical solutions have been studied by several authors, see, for example, [11,19].

In a systematic review of the literature on hybrid differential equations, it is noted that Dhage and Lakshmikantham [5] scrutinized the existence of extremal solutions and a comparison result for first order hybrid differential equation with linear perturbations of the following type:

$$
\frac{d}{d t}\left(\frac{x(t)}{f(t, x(t))}\right)=g(t, x(t)) \text {, a.e. } t \in J, x\left(t_{0}\right)=x_{0} \in \mathbb{R}
$$

where $f \in C(J \times \mathbb{R}, \mathbb{R}-\{0\})$ and $g \in C(J \times \mathbb{R}, \mathbb{R})$. Ge et al. [8] analysed the existence of a mild solution for impulsive hybrid fractional differential equations:

$$
\left.\begin{array}{l}
{ }^{c} D_{0, t}^{q}\left(\frac{u(t)}{f(t, u(t))}\right)=g(t, u(t)), t \in J^{\prime}:=J \backslash\left\{t_{1}, t_{2}, \ldots, t_{m}\right\} \\
u\left(t_{k}^{+}\right)=u\left(t_{k}^{-}\right)+I_{k}\left(u\left(t_{k}^{-}\right)\right), \quad k=1,2, \ldots, m \\
u(0)=u_{0},
\end{array}\right\}
$$

where ${ }^{c} D_{0, t}^{q}$ is the generalized Caputo fractional derivative of order $q \in(0,1)$ with the zero lower limit, $f \in C(J \times \mathbb{R}, \mathbb{R} \backslash\{0\})$ and $g \in C(J \times \mathbb{R}, \mathbb{R})$.

However, to the best of our knowledge, we understand that there has been no previous research made on the oscillation of nonlinear fractional impulsive hybrid partial differential equations. Hence, we believe that we are the first who initiate the oscillation criteria for fractional impulsive partial hybrid differential equations which had not been formerly studied. Motivated by the above observations, we propose the following model of 
the form

$$
\left.\begin{array}{l}
\frac{\partial}{\partial t}\left(r(t) D_{+, t}^{\alpha}\left(\frac{u(x, t)}{h(t, u(x, t))}\right)\right)+q(x, t) g\left(D_{+, t}^{\alpha} u(x, t)\right)= \\
=a(t) \Delta u(x, t)-f\left(t, \int_{0}^{t}(t-s)^{-\alpha} u(x, s) d s\right)+F(x, t), \\
u\left(x, t_{k}^{+}\right)=\gamma_{k}\left(x, t_{k}, u\left(x, t_{k}\right)\right), \\
u_{t}\left(x, t_{k}^{+}\right)=\delta_{k}\left(x, t_{k}, u_{t}\left(x, t_{k}\right)\right), \quad k=1,2, \ldots,(x, t) \in \Omega \times \mathbb{R}_{+} \equiv G .
\end{array}\right\}
$$

where $\Omega$ is a bounded domain in $R^{n}$ with a piecewise smooth boundary $\partial \Omega$, $\alpha \in(0,1)$ is a constant, $D_{+, t}^{\alpha}$ is the Riemann-Liouville fractional derivative of order $\alpha$ of $u$ with respect to $t$, and $\Delta$ is the Laplacian operator in the Euclidean $n$-space $R^{n}$, i. e., $\Delta u(x, t)=\sum_{r=1}^{n} \frac{\partial^{2} u(x, t)}{\partial x_{r}^{2}}$ with the boundary conditions

$$
\frac{\partial u(x, t)}{\partial \eta}+\gamma(x, t) u(x, t)=0, \quad(x, t) \in \partial \Omega \times R_{+}
$$

and

$$
\frac{\partial u(x, t)}{\partial \eta}=0, \quad(x, t) \in \partial \Omega \times R_{+},
$$

where $\eta$ is the unit exterior normal vector to $\partial \Omega$ and $\gamma(x, t)$ is a positive continuous function on $\partial \Omega \times R_{+}$.

Based on the following assumption:

$\left(A_{1}\right) r(t) \in C^{1}\left(R_{+} ; R_{+}\right), r^{\prime}(t) \geqslant 0, a(t) \in P C\left(R_{+} ; R_{+}\right)$;

$\left(A_{2}\right) q(x, t) \in C\left(\bar{G} ; R_{+}\right)$and $q(t)=\min _{x \in \bar{\Omega}} q(x, t)$;

$\left(A_{3}\right) g \in C(R ; R)$ is convex in $R_{+}, u g(u)>0$ and $\frac{g(u)}{u} \geqslant \mu>0$ for $u \neq 0, f(t, x(t)) \in C\left(\left[t_{0}, \infty\right) \times R, R\right)$, there exists a function $p(t) \in C\left(\left[t_{0}, \infty\right), R_{+}\right)$such that $\frac{f(t, K)}{K} \geqslant p(t)$ for $K \neq 0, t \geqslant t_{0} ;$

$\left(A_{4}\right) h \in C\left(R \times\left[t_{0}, \infty\right) ; R-\{0\}\right)$ is convex in $R_{+}$, there exists a function $\zeta(t) \in C\left(\left[t_{0}, \infty\right), R_{+}\right)$such that $h(t, u) \geqslant \xi(x) \zeta(t) \geqslant M \zeta(t), \xi(x)$ is not identically zero on $\left[t_{0}, \infty\right)$ and such that $|\xi(x)| \geqslant M>0$ and $D_{+}^{\alpha} h(t, y)>0$ 
$\left(A_{5}\right) F \in C(\bar{G} ; R)$ such that $\int_{\Omega} F(x, t) d x \leqslant 0 ;$

$\left(A_{6}\right) u(x, t), u_{t}(x, t)$ are piecewise continuous in $t$ with discontinuities of the first kind at $t=t_{k}, k=1,2, \ldots$, and left continuous at $t=t_{k}$, $u\left(x, t_{k}\right)=u\left(x, t_{k}^{-}\right), u_{t}\left(x, t_{k}\right)=u_{t}\left(x, t_{k}^{-}\right), k=1,2, \ldots ;$

$\left(A_{7}\right) \gamma_{k}\left(x, t_{k}, u\left(x, t_{k}\right)\right), \delta_{k}\left(x, t_{k}, u_{t}\left(x, t_{k}\right)\right) \in P C\left(\bar{\Omega} \times R_{+} \times R, R\right), k=1,2, \ldots$ and there exist positive constants $b_{k}, b_{k}^{*}, c_{k}, c_{k}^{*}$ with $c_{k} \leqslant b_{k}^{*}$ such that

$$
b_{k}^{*} \leqslant \frac{\gamma_{k}\left(x, t_{k}, u\left(x, t_{k}\right)\right)}{u\left(x, t_{k}\right)} \leqslant b_{k}, c_{k}^{*} \leqslant \frac{\delta_{k}\left(x, t_{k}, u_{t}\left(x, t_{k}\right)\right)}{u_{t}\left(x, t_{k}\right)} \leqslant c_{k}, k=1,2, \ldots
$$

By a solution of equation (1) and (2)-(3), we mean a nontrivial function $u(x, t) \in C^{1+\alpha}\left(\bar{G}, R_{+}\right)$with

$\int_{0}^{t}(t-s)^{-\alpha} u(x, s) d s \in C^{1}\left(\bar{G}, R_{+}\right), r(t) D_{+, t}^{\alpha}\left(\frac{u(x, t)}{h(t, u(x, t))}\right) \in C^{1}\left(\bar{G}, R_{+}\right)$,

that satisfies $\bar{G}$ and the boundary conditions (2)-(3). A solution of equations (1) and (2)-(3) is called oscillatory if it has arbitrarily large zeros in $G$, and is called nonoscillatory otherwise. Equations (1) and (2)-(3) are said to be oscillatory if all their solutions are oscillatory.

This paper is organized as follows: In Section 2, we present the relevant definitions and lemmas, and in Section 3 we discuss the oscillation problem of (1) subject to the boundary conditions (2)-(3). In Section 4 we present an example to illustrate our main results.

The results in this paper extend and improve numerous findings in the earlier publications that do not include the impulsive effect. We believe that this research work would enable further researchers on the fractional impulsive partial hybrid differential equations.

2. Preliminaries. In this section, we give the fundamental definitions of fractional derivatives and integrals. There are several kinds of definitions of fractional derivatives and integrals. In this paper, we use the Riemann-Liouville left-sided definition on the half-axis $R_{+}$.

Definition 1. [14] The Riemann-Liouville fractional partial derivative of order $0<\alpha<1$ with respect to $t$ of a function $u(x, t)$ is given by

$$
\left(D_{+, t}^{\alpha} y\right)(x, t):=\frac{\partial}{\partial t} \frac{1}{\Gamma(1-\alpha)} \int_{0}^{t}(t-v)^{-\alpha} y(x, v) d v
$$


provided that the right-hand side is pointwise defined on $R_{+}$, where $\Gamma$ is the gamma function.

Definition 2. [14] The Riemann-Liouville fractional integral of order $\alpha>0$ of a function $y: R_{+} \rightarrow R$ on the half-axis $R_{+}$is given by

$$
\left(I_{+}^{\alpha} y\right)(t):=\frac{1}{\Gamma(\alpha)} \int_{0}^{t}(t-v)^{\alpha-1} y(v) d v \quad \text { for } \quad t>0
$$

provided that the right-hand side is pointwise defined on $R_{+}$.

Definition 3. [14] The Riemann-Liouville fractional derivative of order $\alpha>0$ of a function $y: R_{+} \rightarrow R$ on the half-axis $R_{+}$is given by

$$
\left(D_{+}^{\alpha} y\right)(t):=\frac{d^{\lceil\alpha\rceil}}{d t^{\lceil\alpha\rceil}}\left(I_{+}^{\lceil\alpha\rceil-\alpha} y\right)(t) \quad \text { for } \quad t>0
$$

provided that the right-hand side is pointwise defined on $R_{+}$, where $\lceil\alpha\rceil$ is the ceiling function of $\alpha$.

Lemma 1. [14] Let $y(t)$ be a solution of (1) and

$$
E(t)=\int_{0}^{t}(t-\nu)^{-\alpha} y(\nu) d \nu \text { for } \alpha \in(0,1) \text { and } t \geqslant 0 .
$$

Then

$$
E^{\prime}(t)=\Gamma(1-\alpha) D_{+}^{\alpha} y(t) .
$$

Theorem 1. [17] Let $f(x), g(x), h(x) \geqslant 0$ be continuous functions on $[a, b]$ such that

$$
(g(x)-g(y))\left(\frac{f(y)}{h(y)}-\frac{f(x)}{h(x)}\right) \geqslant 0 .
$$

Then the inequality

$$
\frac{\int_{a}^{b} f(x) d x}{\int_{a}^{b} h(x) d x} \geqslant \frac{\int_{a}^{b} f(x) g(x) d x}{\int_{a}^{b} h(x) g(x) d x}
$$


holds. If (9) reverses, then (10) reverses.

Corollary 1. Let $g(x, t), u(x, t), f(t, u(x, t)) \geqslant 0$ be continuous on $\Omega$ and for any fixed $t \in R_{+}$such that

$$
(u(x, t)-u(y, t))\left(\frac{g(y, t)}{f(t, u(y, t))}-\frac{g(x, t)}{f(t, u(x, t))}\right) \geqslant 0 .
$$

Then the inequality

$$
\frac{\int_{\Omega} g(x, t) d x}{\int_{\Omega} f(t, u(x, t)) d x} \geqslant \frac{\int_{\Omega} g(x, t) u(x, t) d x}{\int_{\Omega} f(t, u(x, t)) u(x, t) d x}
$$

holds. If (11) reverses, then (12) reverses.

Proof. We have to prove that

$$
\int_{\Omega} g(x, t) d x \int_{\Omega} f(t, u(x, t)) u(x, t) d x \geqslant \int_{\Omega} f(t, u(x, t)) d x \int_{\Omega} g(x, t) u(x, t) d x,
$$

which is equivalent to

$$
\int_{\Omega} g(x, t) d x \int_{\Omega} f(t, u(y, t)) u(y, t) d y \geqslant \int_{\Omega} f(t, u(x, t)) d x \int_{\Omega} g(y, t) u(y, t) d y,
$$

which implies

$$
\int_{\Omega} \int_{\Omega} u(y, t)(g(x, t) f(t, u(y, t))-g(y, t) f(t, u(x, t))) d x d y \geqslant 0 .
$$

Denote

$$
I=\int_{\Omega} \int_{\Omega} u(y, t)(g(x, t) f(t, u(y, t))-g(y, t) f(t, u(x, t))) d x d y,
$$

then

$$
I=\int_{\Omega} \int_{\Omega} u(x, t)(g(y, t) f(t, u(x, t))-g(x, t) f(t, u(y, t))) d x d y .
$$

Hence 


$$
\begin{array}{r}
2 I=\int_{\Omega} \int_{\Omega}(u(x, t)-u(y, t))(g(y, t) f(t, u(x, t))-g(x, t) f(t, u(y, t))) d x d y= \\
=\int_{\Omega} \int_{\Omega} f(t, u(x, t)) f(t, u(y, t))(u(x, t)-u(y, t)) \times \\
\times\left(\frac{g(y, t)}{f(t, u(y, t))}-\frac{g(x, t)}{f(t, u(x, t))}\right) d x d y
\end{array}
$$

By $(11), 2 I \geqslant 0$.

Remark 1. In particular, replacing $g(x, t)=1$ and $f(t, u(x, t))=$ $=1 / f(t, u(x, t))$ in the above Corollary 1 under the condition

$$
(u(x, t)-u(y, t))(f(t, u(y, t))-f(t, u(x, t))) \geqslant 0,
$$

then

$$
|\Omega| \int_{\Omega} \frac{u(x, t)}{f(t, u(x, t))} d x \geqslant \int_{\Omega} u(x, t) d x \int_{\Omega} \frac{1}{f(t, u(x, t))} d x
$$

holds. If (13) reverses, then (14) reverses.

Remark 2. It is well known that if $f(x)$ is convex and $2 f-f^{2} \geqslant 0$ then $1 / f(x)$ is convex.

3. Main Results. The following notation will be used for convenience:

$$
V(t)=\frac{1}{|\Omega|} \int_{\Omega} u(x, t) d x, \quad|\Omega|=\int_{\Omega} d x .
$$

Throughout the paper, let us assume that $D_{+}^{\alpha} V(t)>0$.

We begin with the following theorem.

Theorem 2. If the fractional differential inequality

$$
\begin{aligned}
& \frac{d}{d t}\left(r(t) D_{+}^{\alpha}\left(\frac{V(t)}{h(t, V(t))}\right)\right)+\mu q(t) D_{+}^{\alpha} V(t)+f(t, E(t)) \leqslant 0, t \neq t_{k}, \\
& b_{k}^{*} \leqslant \frac{V\left(t_{k}^{+}\right)}{V\left(t_{k}\right)} \leqslant b_{k}, \quad c_{k}^{*} \leqslant \frac{V^{\prime}\left(t_{k}^{+}\right)}{V^{\prime}\left(t_{k}\right)} \leqslant c_{k}, k=1,2, \ldots
\end{aligned}
$$

has no eventually positive solution, then every solution of equation (1), (2) is oscillatory in $G$. 
Proof. Assume, for the sake of contradiction, that there is a nonoscillatory solution $u(x, t)$ of (1) and (2), which has a constant sign in the domain $\Omega \times\left[t_{0},+\infty\right)$. With no loss of generality, we assume that $u(x, t)>0$, $(x, t) \in \Omega \times\left[t_{0},+\infty\right), t_{0} \geqslant 0$. Equation (1) is multiplied in both sides by $1 /|\Omega|$ and integrated with respect to $x$ over the domain $\Omega$; we get

$$
\begin{array}{r}
\frac{1}{|\Omega|} \int_{\Omega} \frac{d}{d t}\left(r(t) D_{+, t}^{\alpha}\left(\frac{u(x, t)}{h(t, u(x, t))}\right)\right) d x+\frac{1}{|\Omega|} \int_{\Omega} q(x, t) g\left(D_{+, t}^{\alpha} u(x, t)\right) d x= \\
=\frac{1}{|\Omega|} \int_{\Omega} a(t) \Delta u(x, t) d x-\frac{1}{|\Omega|} \int_{\Omega} f\left(t, \int_{0}^{t}(t-s)^{-\alpha} u(x, s) d s\right) d x+ \\
\quad+\frac{1}{|\Omega|} \int_{\Omega} F(x, t) d x
\end{array}
$$

From Green's formula and the Robin boundary condition (2), we get

$$
\int_{\Omega} \Delta u(x, t) d x=\int_{\partial \Omega} \frac{\partial u(x, t)}{\partial \eta} d S=-\int_{\partial \Omega} \gamma(x, t) u(x, t) d S \leqslant 0,
$$

where $d S$ is the surface element on $\partial \Omega$. From $\left(A_{2}\right),\left(A_{3}\right)$ and Jensen's inequality, we have

$$
\begin{aligned}
\frac{1}{|\Omega|} \int_{\Omega} q(x, t) g\left(D_{+, t}^{\alpha} u(x, t)\right) d x & \geqslant q(t) \frac{1}{|\Omega|} \int_{\Omega} g\left(D_{+, t}^{\alpha} u(x, t)\right) d x \geqslant \\
& \geqslant q(t) g\left(D_{+}^{\alpha} V(t)\right) \geqslant \mu q(t) D_{+}^{\alpha} V(t) .
\end{aligned}
$$

Again applying Jensen's inequality and using Lemma 1,

$$
\begin{array}{r}
\frac{1}{|\Omega|} \int_{\Omega} f\left(t, \int_{0}^{t}(t-s)^{-\alpha} u(x, s) d s\right) d x \geqslant f\left(t, \frac{1}{|\Omega|} \int_{\Omega} \int_{0}^{t}(t-s)^{-\alpha} u(x, s) d s d x\right) \\
=f\left(t, \int_{0}^{t}(t-s)^{-\alpha} V(s) d s\right)=f(t, E(t)) . \quad
\end{array}
$$

Now applying Remark 1 and 2, 


$$
\begin{aligned}
& \frac{1}{|\Omega|} \int_{\Omega} \frac{d}{d t}\left(r(t) D_{+, t}^{\alpha}\left(\frac{u(x, t)}{h(t, u(x, t))}\right)\right) d x \geqslant \\
& \geqslant \frac{d}{d t}\left(r(t) D_{+}^{\alpha}\left(\frac{1}{|\Omega|} \int_{\Omega} \frac{u(x, t)}{h(t, u(x, t))}\right)\right) d x \geqslant \frac{d}{d t}\left(r(t) D_{+}^{\alpha}\left(\frac{V(t)}{h(t, V(t))}\right)\right) .
\end{aligned}
$$

In view of $(17)-(21)$ and $\left(A_{4}\right)$ yield

$$
\frac{d}{d t}\left(r(t) D_{+}^{\alpha}\left(\frac{V(t)}{h(t, V(t))}\right)\right)+\mu q(t) D_{+}^{\alpha} V(t)+f(t, E(t)) \leqslant 0, \quad t \neq t_{k} .
$$

For $t \geqslant t_{0}, t=t_{k}, k=1,2, \ldots$, multiplying both sides of $(1)$ by $\frac{1}{|\Omega|}$ and integrating with respect to $x$ over the domain $\Omega$, we obtain

$$
b_{k}^{*} \leqslant \frac{u\left(x, t_{k}^{+}\right)}{u\left(x, t_{k}\right)} \leqslant b_{k}, \quad c_{k}^{*} \leqslant \frac{u_{t}\left(x, t_{k}^{+}\right)}{u_{t}\left(x, t_{k}\right)} \leqslant c_{k} .
$$

According to (15), we get

$$
b_{k}^{*} \leqslant \frac{V\left(t_{k}^{+}\right)}{V\left(t_{k}\right)} \leqslant b_{k}, \quad c_{k}^{*} \leqslant \frac{V^{\prime}\left(t_{k}^{+}\right)}{V^{\prime}\left(t_{k}\right)} \leqslant c_{k}, k=1,2, \ldots
$$

Hence, $V(t)$ is a positive solution of impulsive differential inequality (16).

Theorem 3. If there exists a positive function $\phi \in C^{1}\left([0, \infty) ; R_{+}\right)$and

$$
\int_{t_{0}}^{+\infty} \prod_{t_{0} \leqslant t_{k}<s}\left(\frac{c_{k}}{b_{k}^{*}}\right)^{-1}\left(p(s) \phi(s)-\frac{1}{4 M \Gamma(1-\alpha)} \frac{\left(\phi^{\prime}(s)\right)^{2}}{\phi(s)} \frac{r(s)}{\zeta(s)}\right) d s=+\infty,
$$

holds, then every solution of boundary value problem (1)-(2) is oscillatory in $G$.

Proof. Assume that there exists a nonoscillatory solution $u(x, t)$ of (1)-(2) if the conditions of Theorem 3 hold. Suppose that $V(t)>0$ is a solution of inequality $(16)$ and $D_{+}^{\alpha} V(t)>0$. By $\left(A_{3}\right)$, inequality (16) can be reduced to the following form

$$
\left.\frac{d}{d t}\left(r(t) D_{+}^{\alpha}\left(\frac{V(t)}{h(t, V(t))}\right)\right)+\mu q(t) D_{+}^{\alpha} V(t)+p(t) E(t)\right) \leqslant 0, t \neq t_{k} .
$$


Define

$$
Z(t)=\phi(t) \frac{r(t) D_{+}^{\alpha}\left(\frac{V(t)}{h(t, V(t))}\right)}{E(t)}, t \geqslant t_{1}
$$

Then $Z(t)>0$, since $E(t)>0$ for $t \geqslant t_{1}$. Differentiating (24) and using (16) and Lemma 1, we have

$$
\begin{aligned}
Z^{\prime}(t) & \leqslant \frac{\phi^{\prime}(t)}{\phi(t)} Z(t)-\mu q(t) \phi(t) \frac{D_{+}^{\alpha} V(t)}{E(t)}-p(t) \phi(t)-Z(t) \frac{E^{\prime}(t)}{E(t)} \leqslant \\
& \leqslant \frac{\phi^{\prime}(t)}{\phi(t)} Z(t)-p(t) \phi(t)-Z^{2}(t) \frac{E^{\prime}(t)}{\phi(t) r(t) D_{+}^{\alpha}\left(\frac{V(t)}{h(t, V(t))}\right)} \leqslant \\
& \leqslant \frac{\phi^{\prime}(t)}{\phi(t)} Z(t)-p(t) \phi(t)-\Gamma(1-\alpha) \frac{h(t, V(t))}{\phi(t) r(t)} Z^{2}(t) .
\end{aligned}
$$

By $\left(A_{4}\right)$, we get

$$
\begin{aligned}
Z^{\prime}(t) & \leqslant \frac{\phi^{\prime}(t)}{\phi(t)} Z(t)-p(t) \phi(t)-M \Gamma(1-\alpha) \frac{\zeta(t)}{\phi(t) r(t)} Z^{2}(t), \\
Z\left(t_{k}^{+}\right) & \leqslant \frac{c_{k}}{b_{k}^{*}} Z\left(t_{k}\right) .
\end{aligned}
$$

Define

$$
\Theta(t)=\prod_{t_{0} \leqslant t_{k}<t}\left(\frac{c_{k}}{b_{k}^{*}}\right)^{-1} Z(t)
$$

In fact, $Z(t)$ is continuous on each interval $\left(t_{k}, t_{k+1}\right]$ and in consideration of (25). It follows that for $t \geqslant t_{0}$,

$$
\Theta\left(t_{k}^{+}\right)=\prod_{t_{0} \leqslant t_{i} \leqslant t_{k}}\left(\frac{c_{k}}{b_{k}^{*}}\right)^{-1} Z\left(t_{k}^{+}\right) \leqslant \prod_{t_{0} \leqslant t_{i}<t_{k}}\left(\frac{c_{k}}{b_{k}^{*}}\right)^{-1} Z\left(t_{k}\right)=\Theta\left(t_{k}\right),
$$

and for all $t \geqslant t_{0}$,

$$
\Theta\left(t_{k}^{-}\right)=\prod_{t_{0} \leqslant t_{i} \leqslant t_{k-1}}\left(\frac{c_{k}}{b_{k}^{*}}\right)^{-1} Z\left(t_{k}^{-}\right) \leqslant \prod_{t_{0} \leqslant t_{i}<t_{k}}\left(\frac{c_{k}}{b_{k}^{*}}\right)^{-1} Z\left(t_{k}\right)=\Theta\left(t_{k}\right),
$$


which implies that $\Theta(t)$ is continuous on $\left[t_{0},+\infty\right)$.

$$
\begin{gathered}
\Theta^{\prime}(t)+M \Gamma(1-\alpha) \prod_{t_{0} \leqslant t_{k}<t}\left(\frac{c_{k}}{b_{k}^{*}}\right) \frac{\zeta(t)}{\phi(t) r(t)} \Theta^{2}(t)+\prod_{t_{0} \leqslant t_{k}<t}\left(\frac{c_{k}}{b_{k}^{*}}\right)^{-1} p(t) \phi(t)- \\
-\frac{\phi^{\prime}(t)}{\phi(t)} \Theta(t)=\prod_{t_{0} \leqslant t_{k}<t}\left(\frac{c_{k}}{b_{k}^{*}}\right)^{-1} Z^{\prime}(t)-\prod_{t_{0} \leqslant t_{i}<t_{k}}\left(\frac{c_{k}}{b_{k}^{*}}\right)^{-1} \frac{\phi^{\prime}(t)}{\phi(t)} \Theta(t)+ \\
+\prod_{t_{0} \leqslant t_{k}<t}\left(\frac{c_{k}}{b_{k}^{*}}\right) \prod_{t_{0} \leqslant t_{k}<t}\left(\frac{c_{k}}{b_{k}^{*}}\right)^{-2} \Gamma(1-\alpha) \frac{M \zeta(t) \Theta^{2}(t)}{\phi(t) r(t)}+\prod_{t_{0} \leqslant t_{i}<t_{k}}\left(\frac{c_{k}}{b_{k}^{*}}\right)^{-1} p(t) \phi(t)= \\
=\prod_{t_{0} \leqslant t_{k}<t}\left(\frac{c_{k}}{b_{k}^{*}}\right)^{-1}\left[Z^{\prime}(t)-\frac{\phi^{\prime}(t)}{\phi(t)} Z(t)+p(t) \phi(t)+M \Gamma(1-\alpha) \frac{\zeta(t)}{\phi(t) r(t)} Z^{2}(t)\right] \leqslant 0 .
\end{gathered}
$$

That is,

$$
\begin{aligned}
\Theta^{\prime}(t) \leqslant-M \Gamma(1-\alpha) \prod_{t_{0} \leqslant t_{k}<t}\left(\frac{c_{k}}{b_{k}^{*}}\right) \frac{\zeta(t)}{\phi(t) r(t)} \Theta^{2}(t)- & \\
& -\prod_{t_{0} \leqslant t_{k}<t}\left(\frac{c_{k}}{b_{k}^{*}}\right)^{-1} p(t) \phi(t)+\frac{\phi^{\prime}(t)}{\phi(t)} \Theta(t) .
\end{aligned}
$$

Then, using the inequality, $\lambda A B^{\lambda-1}-A^{\lambda} \leqslant(\lambda-1) B^{\lambda}, \lambda \geqslant 1$, we have

$$
\Theta^{\prime}(t) \leqslant-\prod_{t_{0} \leqslant t_{k}<t}\left(\frac{c_{k}}{b_{k}^{*}}\right)^{-1}\left[p(t) \phi(t)-\frac{1}{4 M \Gamma(1-\alpha)} \frac{\left(\phi^{\prime}(t)\right)^{2}}{\phi(t)} \frac{r(t)}{\zeta(t)}\right] .
$$

Integrating the last inequality from $t_{0}$ to $t$, which yields

$$
\Theta(t) \leqslant \Theta\left(t_{0}\right)-\int_{t_{0}}^{t} \prod_{t_{0} \leqslant t_{k}<s}\left(\frac{c_{k}}{b_{k}^{*}}\right)^{-1}\left[p(s) \phi(s)-\frac{1}{4 M \Gamma(1-\alpha)} \frac{\left(\phi^{\prime}(s)\right)^{2}}{\phi(s)} \frac{r(s)}{\zeta(s)}\right] d s .
$$

Letting $t \rightarrow+\infty$, we get a contradiction to the hypothesis (22).

Theorem 4. Assume that there exist functions $\phi(t)$ and $\psi(t) \in C^{1}([0, \infty)$; $(0,+\infty))$ in which $\phi(t)$ is nondecreasing with respect to $t$ and the functions $H(t, s), h(t, s) \in C^{1}(D, R)$ in which $D=\left\{(t, s) \mid t \geqslant s \geqslant t_{0}>0\right\}$, such that $\left(T_{1}\right) H(t, t)=0$ for $t \geqslant t_{0}$ and $H(t, s)>0$ for $t>s \geqslant t_{0}$;

$\left(T_{2}\right) H_{t}^{\prime}(t, s) \geqslant 0, H_{s}^{\prime}(t, s) \leqslant 0$; 
$\left(T_{3}\right) h(t, s)=-\frac{\partial H(t, s)}{\partial s} \psi(s)-H(t, s) \psi^{\prime}(s)-H(t, s) \psi(s) \frac{\phi^{\prime}(s)}{\phi(s)}$.

If

$$
\begin{aligned}
\limsup _{t \rightarrow+\infty} \int_{t_{0}}^{t} \prod_{t_{0} \leqslant t_{k}<s}\left(\frac{c_{k}}{b_{k}^{*}}\right)^{-1}(p(s) \phi(s) H(t, s) \psi(s)- & \\
& \left.-\frac{1}{4 M \Gamma(1-\alpha)} \frac{h^{2}(t, s) \Psi(s)}{H(t, s) \psi(s)}\right) d s=\infty
\end{aligned}
$$

where $\Psi(s)=\frac{\phi(s) r(s)}{\zeta(s)}$, then each solution of (1)-(2) is oscillatory in $G$.

Proof. We prove that the equation (26) has no eventually positive solutions if the conditions of Theorem 4 hold. As in the proof of Theorem 3, we obtain

$$
\begin{aligned}
\Theta^{\prime}(t) \leqslant & -M \Gamma(1-\alpha) \prod_{t_{0} \leqslant t_{k}<t}\left(\frac{c_{k}}{b_{k}^{*}}\right) \frac{\zeta(t)}{\phi(t) r(t)} \Theta^{2}(t)- \\
& -\prod_{t_{0} \leqslant t_{k}<t}\left(\frac{c_{k}}{b_{k}^{*}}\right)^{-1} p(t) \phi(t)+\frac{\phi^{\prime}(t)}{\phi(t)} \Theta(t) .
\end{aligned}
$$

Multiplying the above inequality by $H(t, s) \psi(s)$ for $t \geqslant s \geqslant T$ and integrating from $T$ to $t$, we have

$$
\begin{aligned}
\int_{T}^{t} H(t, s) & \psi(s) \Theta^{\prime}(s) d s \leqslant-\int_{T}^{t} \prod_{t_{0} \leqslant t_{k}<s}\left(\frac{c_{k}}{b_{k}^{*}}\right)^{-1} H(t, s) \psi(s) p(s) \phi(s) d s- \\
& -M \Gamma(1-\alpha) \int_{T}^{t} \prod_{t_{0} \leqslant t_{k}<s}\left(\frac{c_{k}}{b_{k}^{*}}\right) H(t, s) \psi(s) \frac{\zeta(s)}{\phi(s) r(s)} \Theta^{2}(s) d s+ \\
& +\int_{T}^{t} H(t, s) \psi(s) \frac{\phi^{\prime}(s)}{\phi(s)} \Theta(s) d s .
\end{aligned}
$$

Thus

$\int_{T}^{t} \prod_{t_{0} \leqslant t_{k}<s}\left(\frac{c_{k}}{b_{k}^{*}}\right)^{-1} H(t, s) \psi(s) p(s) \phi(s) d s \leqslant H(t, T) \psi(T) \Theta(T)-$ 


$$
\begin{aligned}
& -\int_{T}^{t}\left[-\frac{\partial H(t, s)}{\partial s} \psi(s)-H(t, s) \psi^{\prime}(s)-H(t, s) \psi(s) \frac{\phi^{\prime}(s)}{\phi(s)}\right] d s- \\
& -M \Gamma(1-\alpha) \int_{T}^{t} \prod_{t_{0} \leqslant t_{k}<s}\left(\frac{c_{k}}{b_{k}^{*}}\right) H(t, s) \psi(s) \frac{\zeta(s)}{\phi(s) r(s)} \Theta^{2}(s) d s,
\end{aligned}
$$

which implies that

$$
\begin{aligned}
& \int_{T}^{t} \prod_{t_{0} \leqslant t_{k}<s}\left(\frac{c_{k}}{b_{k}^{*}}\right)^{-1}[H(t, s) \psi(s) p(s) \phi(s) d s- \\
& \left.\quad-\frac{1}{4 M \Gamma(1-\alpha)} \frac{h^{2}(t, s) \phi(s) r(s)}{\zeta(s) H(t, s) \psi(s)}\right] d s \leqslant H(t, T) \psi(T) \Theta(T),
\end{aligned}
$$

from (27), for $t \geqslant T \geqslant t_{0}$, we have

$$
\begin{gathered}
\frac{1}{H\left(t, t_{0}\right)} \int_{T}^{t} \prod_{t_{0} \leqslant t_{k}<s}\left(\frac{c_{k}}{b_{k}^{*}}\right)^{-1}[H(t, s) \psi(s) p(s) \phi(s)- \\
\left.-\frac{1}{4 M \Gamma(1-\alpha)} \frac{h^{2}(t, s) \phi(s) r(s)}{\zeta(s) H(t, s) \psi(s)}\right] d s= \\
=\frac{1}{H\left(t, t_{0}\right)}\left[\int_{t_{0}}^{t}+\int_{T}^{t}\right]\left\{\prod_{t_{0} \leqslant t_{k}<s}\left(\frac{c_{k}}{b_{k}^{*}}\right)^{-1}[H(t, s) \psi(s) p(s) \phi(s)-\right. \\
\left.\left.\quad-\frac{1}{4 M \Gamma(1-\alpha)} \frac{h^{2}(t, s) \phi(s) r(s)}{\zeta(s) H(t, s) \psi(s)}\right]\right\} d s \leqslant \\
\leqslant \int_{t_{0}}^{T} \prod_{t_{0} \leqslant t_{k}<s}\left(\frac{c_{k}}{b_{k}^{*}}\right)^{-1} \psi(s) p(s) \phi(s) d s+\psi(T) \Theta(T) .
\end{gathered}
$$

Letting $t \rightarrow+\infty$, we have

$$
\begin{aligned}
\limsup _{t \rightarrow+\infty} \frac{1}{H\left(t, t_{0}\right)} \int_{t_{0}}^{t} \prod_{t_{0} \leqslant t_{k}<s}\left(\frac{c_{k}}{b_{k}^{*}}\right)^{-1}[H(t, s) \psi(s) p(s) \phi(s)- & \\
& \left.-\frac{1}{4 M \Gamma(1-\alpha)} \frac{h^{2}(t, s) \Psi(s)}{H(t, s) \psi(s)}\right] d s \leqslant
\end{aligned}
$$




$$
\leqslant \int_{t_{0}}^{T} \prod_{t_{0} \leqslant t_{k}<s}\left(\frac{c_{k}}{b_{k}^{*}}\right)^{-1} \psi(s) p(s) \phi(s) d s+\psi(T) \Theta(T)<\infty
$$

which leads to a contradiction with (26).

Remark 3. In Theorem 4, by choosing $\phi(t)=\psi(t)=1$, we have the following result.

Corollary 1. Assume that the conditions of Theorem 4 hold, and

$$
\limsup _{t \rightarrow+\infty} \int_{t_{0}}^{t} \prod_{t_{0} \leqslant t_{k}<s}\left(\frac{c_{k}}{b_{k}^{*}}\right)^{-1}\left(p(s) H(t, s)-\frac{1}{4 M \Gamma(1-\alpha)} \frac{h^{2}(t, s) \Psi(s)}{H(t, s) \zeta(s)}\right) d s=\infty
$$

where $h(t, s)=-\frac{\partial H(t, s)}{\partial s}$, then each solution of $(1),(2)$ is oscillatory.

Remark 4. All the above theorems and corollaries are true for the equation (1) with the boundary condition (3).

Remark 5. The results obtained in this paper can be extended for the more general differential equations having a damped term with a delay of the form

$$
\begin{aligned}
& \frac{\partial}{\partial t}\left(r(t) D_{+, t}^{\alpha}\left(\frac{u(x, t)}{f(t, u(x, t))}\right)\right)+q(x, t) g\left(D_{+, t}^{\alpha}\left(\frac{u(x, t)}{f(t, u(x, t))}\right)\right)+ \\
& +r(t) u(x, \sigma(t))+h\left(\int_{0}^{t}(t-\xi)^{-\alpha} u(x, \xi) d \xi\right)=a(t) \Delta u(x, t)+F(x, t), \\
& u\left(x, t_{k}^{+}\right)=\gamma_{k}\left(x, t_{k}, u\left(x, t_{k}\right)\right), \\
& u_{t}\left(x, t_{k}^{+}\right)=\delta_{k}\left(x, t_{k}, u_{t}\left(x, t_{k}\right)\right), \quad k=1,2, \ldots, \quad(x, t) \in \Omega \times R_{+}
\end{aligned}
$$

4. Examples. In this section, we give examples to illustrate our main results.

Example 1. Consider the fractional nonlinear partial hybrid differen- 
tial equations

$$
\begin{aligned}
& \frac{\partial}{\partial t}\left(\sqrt{2} D_{+, t}^{\frac{1}{2}}\left(\frac{u(x, t)}{e^{-t}}\right)\right)+D_{+, t}^{\frac{1}{2}} u(x, t)=e^{t} \Delta u(x, t)- \\
& -\frac{1}{\sqrt{2 \pi}(\cos t C(x)+\sin t S(x))} \int_{0}^{t}(t-s)^{-\frac{1}{2}} u(x, s) d s+F(x, t), t \neq t_{k}, \\
& u\left(x, t_{k}^{+}\right)=\frac{k}{k+1}\left(x, t_{k}, u\left(x, t_{k}\right)\right) \\
& u_{t}\left(x, t_{k}^{+}\right)=u_{t}\left(x, t_{k}, u_{t}\left(x, t_{k}\right)\right), \quad k=1,2, \ldots
\end{aligned}
$$

for $(x, t) \in(0, \pi) \times[0,+\infty)=G$, with the boundary condition

$$
\frac{\partial}{\partial x} u(0, t)=\frac{\partial}{\partial x} u(\pi, t)=0 .
$$

Here $\alpha=\frac{1}{2}, r(t)=\sqrt{2}, a(t)=e^{t}, h(t, u)=e^{-t}, \zeta(t)=e^{-t}, q(t)=1$, $b_{k}=b_{k}^{*}=1, c_{k}=c_{k}^{*}=\frac{k+1}{k}, p(t)=1 /(\sqrt{2 \pi}(\cos t C(x)+\sin t S(x)))$ where $C(x)$ and $S(x)$ are the Fresnel integrals, namely

$$
C(x)=\int_{0}^{x} \cos \left(\frac{1}{2} \pi t^{2}\right) d t, S(x)=\int_{0}^{x} \sin \left(\frac{1}{2} \pi t^{2}\right) d t
$$

with $|C(x)| \leqslant \pi,|S(x)| \leqslant \pi, g(u)=u, \mu=1, M=1$ and

$$
F(x, t)=\cos x \widehat{C}_{t}\left(\frac{1}{2},-1,1\right)+\sqrt{\frac{t}{\pi}}-1-\cos x \sin t
$$

where

$$
\widehat{C}_{t}\left(\frac{1}{2},-1,1\right)=\frac{e^{-t}}{\Gamma\left(\frac{1}{2}\right)} \int_{0}^{t} s^{-\frac{1}{2}} e^{-s} \cos (t-s) d s
$$

It is easy to see that $D^{\frac{1}{2}} e^{-t}=e^{-t}\left(\sqrt{\frac{\pi}{t}}-1\right)>0$ if $t<\pi$.

If we take $\phi(t)=1$ then $\phi^{\prime}(t)=0$, since $t_{0}=1, t_{k}=2^{k}$. Consider

$$
\int_{t_{0}}^{+\infty} \prod_{t_{0} \leqslant t_{k}<s}\left(\frac{c_{k}}{b_{k}^{*}}\right)^{-1}\left(p(s) \phi(s)-\frac{1}{4 M \Gamma(1-\alpha)} \frac{\left(\phi^{\prime}(s)\right)^{2}}{\phi(s)} \frac{r(s)}{\zeta(s)}\right) d s=
$$




$$
\begin{aligned}
& =\lim _{t \rightarrow+\infty} \int_{t_{0}}^{t} \prod_{t_{0} \leqslant t_{k}<s} \frac{k}{k+1} \frac{1}{\sqrt{2 \pi}(\cos s C(x)+\sin s S(x))} d s \geqslant \\
& \geqslant(2 \pi)^{\frac{3}{2}} \int_{1}^{+\infty} \prod_{1<t_{k}<s} \frac{k}{k+1} d s= \\
& =(2 \pi)^{\frac{3}{2}}\left(\int_{1}^{t_{1}} \prod_{1<t_{k}<s} \frac{k}{k+1} d s+\int_{t_{1}^{+}} \prod_{1<t_{k}<s} \frac{k}{k+1} d s+\ldots\right)= \\
& =(2 \pi)^{\frac{3}{2}}\left(1+\frac{1}{2} \times 2+\frac{1}{2} \times \frac{2}{3} \times 2^{2}+\ldots\right)=(2 \pi)^{\frac{3}{2}} \sum_{n=0}^{+\infty} \frac{2^{n}}{n+1}=+\infty .
\end{aligned}
$$

Thus, all the conditions of Theorem 3 are satisfied. Hence, every solution of (29) and (30) is oscillatory. In fact, $u(x, t)=\cos x e^{-t} \cos t$ is one such solution of (29)-(30).

5. Conclusion. In this article, we have identified some new sufficient conditions for all solutions of fractional impulsive partial differential equations to be oscillatory, which has a scope beyond the available results in the existing literature. Required example has also been incorporated in the paper for the confirmation of the results.

\section{References}

[1] Abbas S., Benchora M., N'Guerekata G. M. Topics in fractional differential equations, Springer, Newyork, 2012.

[2] Bainov D. D., Mishev D. P. Estimates of solutions of impulsive parabolic equations and applications to the population dynamics, Publ. Math., 1996, vol. 40, pp. $85-94$.

[3] Chatzarakis G. E., Sadhasivam V., Raja T., Kalaimani T. Oscillation of certain nonlinear impulsive neutral partial differential equations with continuous distributed deviating arguments and a damping term, Dyn. Contin. Discrete Impulsive Syst. Ser. A Math. Anal., 2018, vol. 25, pp. 329-345.

[4] Daftardar-Gejji V. Fractional calculus theory and applications, Narosa publishing house Pvt. Ltd., 2014.

[5] Dhage B. C., Lakshmikantham V. Basic results on hybrid differential equations, Nonlinear Anal. Hybrid syst., 2010, vol. 4, pp. 414-424.

[6] Erbe L., Freedman H., Liu X. Z., Wu J. H. Comparison principles for impulsive parabolic equations with application to models of single species growth, J. Aust. Math. Soc., 1991, vol. 32, pp. 382-400. 
[7] Feng Q., Meng F. Oscillation of solutions to nonlinear forced fractional differential equations, Electron. J. Differential Equations, 2013, vol. 169, pp. $1-10$.

[8] Ge H., Xin J. On the existence of a mild solution for impulsive hybrid fractional differential equations, Adv. Difference Equ., 2013, vol. 211.

[9] Gopalsamy K., Zhang B. G. On delay differential equations with impulses, J. Math. Anal. Appl., 1989, vol. 139, pp. 110-122.

[10] Herzallah M. A. E., Baleanu D. On fractional order hybrid differential equations, Abstr. Appl. Anal., Hindawi, 2014, Article ID 389386, pp. 1-7.

[11] Heydari M., Loghmani G. B., Hosseini S. M., Karbassi S. M., Application of hybrid functions for solving diffing-harmonic oscillator, J. Difference Equ., Hindawi, 2014, Article ID 210754, pp. 1-9.

[12] Jiang G., Lu Q. Impulsive state feedback control of a predator-prey model, J. Comput. Appl. Math., 2007, vol. 200, no. 1, pp. 193-207.

[13] Kalaimani T., Raja T., Sadhasivam V., Saker S. H. Oscillation of impulsive neutral partial differential equations with distributed deviating arguments, Bull. Math. Soc. Sci. Math. Roumanie, Tome 61, 2018, vol. 109, no. 1, pp. $51-68$.

[14] Kilbas A. A., Srivastava H. M., Trujillo J. J. Theory and applications of fractional differential equations, Elsevier science, B.V. Amsterdam, 2006.

[15] Lakshmikantham V., Bainov D. D., Simenov P. S. Theory of impulsive differential equations, World scientific publishers, Singapore, 1989.

[16] Li W. N., Sheng W. Oscillation properties for solutions of a kind of partial fractional differential equations with damping term, J. Nonlinear Sci Appl., 2016, pp. $1600-1608$.

[17] Liu W., Ngo Q. A., Huy V. N. Several interesting integral inequalities, J. Math. Inequal., 2009, vol. 3, no. 2, pp. 201-212.

[18] Mainardi F. Fractional calculus and waves in linear viscoelasticity, Imberical college press, London, 2010.

[19] Maleknejad K., Torkzadeh L. Application of hybrid functions for solving oscillator equations, Rom. Journ. Phys., 2015, vol. 60, no. 1-2, pp. 87-98.

[20] Prakash P., Harikrishnan S. Oscillation of solutions of impulsive vector hyperbolic differential equations with delays, Appl. Anal., 2012, vol. 91, no. 3, pp. $459-473$.

[21] Prakash P., Harikrishnan S., Nieto J.J., Kim J. H. Oscillation of a time fractional partial differential equations, Electron. J. Qual. Theory Differ. Equ., 2014, vol. 15, pp. 1-10. 
[22] Sadhasivam V., Kavitha J. Forced oscillation of fractional order partial differential equations with damping and functional arguments, IJPAM, 2016, vol. 106, no. 7, pp. 89-107.

[23] Sadhasivam V., Kavitha J., Raja T. Forced oscillation of impulsive neutral hyperbolic differential equations, International journal of applied engineering research, 2016, vol. 11, no. 1, pp. 58-63.

[24] Sadhasivam V., Kavitha J., Deepa M. On the oscillation of nonlinear fractional partial differential equations, Global JPAM, 2017, vol. 13, no. 5, pp. $223-231$.

[25] Sadhasivam V., Raja T., Kalaimani T. Oscillation of system of impulsive neutral partial differential equations with damping term, IJPAM, 2017, vol. 115 , no. 9 , pp. $65-81$.

[26] Samko S. G., Kilbas A. A., Marichev O. I. Fractional integrals and derivatives, Gordon and breach science publishers, Yverdon, 1993.

[27] Sitho S., Ntouyas S. K., Tariboon J. Existence results for hybrid fractional integro-differential equations, Bound. Value Probl., 2015, vol. 113, pp.113.

[28] Vasundara Devi J., Giribabu N. On hybrid Caputo fractional differential equations with variable moments of impulse, Eur. J. Pure Appl. Math., 2014 , vol. 7 , no. 2 , pp. $115-128$.

[29] $\mathrm{Wu}$ J. Theory and applications of partial functional differential equations, Springer, Newyork, 1996.

[30] Yang J., Liu A., Liu G. Oscillation of solutions to neutral nonlinear impulsive hyperbolic equations with several delays, Electron. J. Differ. Eq., 2013, vol. 27, pp. 1-10.

[31] Yoshida N. Oscillation theory of partial differential equations, World Scientific, Singapore, 2008.

[32] Zhao Y., Sun S., Han Z., Li Q. Theory of fractional hybrid differential equations, Computers and mathematics with applications, 2011, vol. 62, pp. $1312-1324$.

[33] Zhou Y. Basic theory of fractional differential equations, World Scientific, Singapore, 2010.

Received January 14, 2019.

In revised form, January 22, 2019.

Accepted June 10, 2019.

Published online June 15, 2019. 


\section{Sadhasivam}

Post Graduate and Research Department of Mathematics, Thiruvalluvar Government Arts College (Affli. to Periyar University, Salem), Rasipuram - 637 401, Namakkal Dt., Tamil Nadu, India.

E-mail: ovsadha@gmail.com

\section{Deepa}

Department of Mathematics, Pavai Arts and Science College for Women, Anaipalayam, Rasipuram - 637401, Namakkal Dt., Tamil Nadu, India. E-mail: mdeepa.maths@gmail.com 